\title{
Methodology used to develop the AANS/CNS management of brain metastases evidence-based clinical practice parameter guidelines
}

\author{
Paula D. Robinson • Steven N. Kalkanis • \\ Mark E. Linskey $\cdot$ P. Lina Santaguida
}

Received: 7 September 2009/Accepted: 8 November 2009/Published online: 3 December 2009

(C) The Author(s) 2009. This article is published with open access at Springerlink.com

\section{Background}

Brain metastases, which occur in approximately $20-40 \%$ of individuals with systemic cancer, represent a significant cause of morbidity and mortality and overwhelm all other types of brain tumors in terms of incidence and public health impact [1]. Considerable research has focused on improving survival and quality of life for this patient population. Given the expanding knowledge base and the rapid emergence of new therapies, the American Association of Neurological Surgeons (AANS), the Congress of Neurological Surgeons (CNS), and the AANS/CNS Joint Tumor Section jointly funded an initiative to produce methodologically rigorous evidence-linked clinical practice parameter guidelines on this topic. The overall objective of this series of guideline papers is to provide the latest up-to-date evidence-based recommendations for the management of patients with brain metastases centering on eight questions related to commonly encountered clinical scenarios (Tables 1, 2, 3). Accomplishment of this goal required undertaking a systematic review of the literature.

The McMaster University Evidence-based Practice Center (EPC), which is an academic research unit partially funded by an EPC grant from the Agency for Healthcare

P. D. Robinson - P. L. Santaguida

McMaster University Evidence-based Practice Center,

Hamilton, ON, Canada

S. N. Kalkanis $(\bowtie)$

Department of Neurosurgery, Henry Ford Health System,

2799 West Grand Blvd, K-11, Detroit, MI 48202, USA

e-mail: kalkanis@neuro.hfh.edu; skalkan1@hfhs.org

M. E. Linskey

Department of Neurosurgery, University of California-Irvine

Medical Center, Orange, CA, USA
Research and Quality (AHRQ), with specialized expertise in evidence-based medicine and the development of systematic reviews, was contracted to performed the systematic review in consultation with the guideline panel assembled for the initiative. The McMaster EPC also served as facilitators during the guideline development, consensus and writing processes.

The Joint Tumor Section of the AANS/CNS recruited representatives from surgical neuro-oncology (including microsurgical, stereotactic radiosurgery and experimental therapies), radiation oncology (fractionated radiotherapy, stereotactic radiosurgery and brachytherapy) and medical neuro-oncology (chemotherapy and experimental therapies) to form a multi-disciplinary panel of 17 clinical experts who developed the evidence-based practice guidelines from the systematic review results (Table 4). These seventeen experts across several disciplines were all nominated and selected by the Executive Committee of the AANS/CNS Tumor Section based on their clinical expertise and recognized contributions to the field of neurooncology in general and brain metastases in particular. The Tumor Section Executive Committee then selected a chairperson for this endeavor to organize and lead the effort, serving also to encourage and manage debate on the various topics involved.

\section{Scope of the systematic review and clinical practice guidelines}

The specific questions regarding the treatment of brain metastases addressed by the systematic review and the resulting practice guidelines were determined collaboratively by the clinical guideline panel and methodologists at the McMaster EPC. In total, eight questions were agreed 
Table 1 Guideline questions specifically addressing treatment of patients with newly diagnosed brain metastases

For patients with newly diagnosed brain metastases

Should whole-brain radiation therapy (WBRT) be used as the sole therapy in patients with newly diagnosed, surgically accessible, single brain metastases, compared with WBRT plus surgical resection, and in what clinical settings?

Comparisons include:

- WBRT vs. WBRT + surgical resection

If WBRT is used, is there an optimal dosing/fractionation schedule? Comparisons include:

- Dose/fractionation schedule 1 vs. dose/fractionation schedule 2

If WBRT is used, what impact does tumor histopathology have on treatment outcomes?

- Any study evaluating the outcome of WBRT by tumor histopathology (e.g., radiation-sensitive tumors vs. radiationresistant tumors) in patients with newly diagnosed metastatic brain tumors

Should patients with newly diagnosed metastatic brain tumors undergo open surgical resection versus radiosurgery or other treatment modalities, and in what clinical settings?

Comparisons include:

- Surgical resection vs. WBRT

- Surgical resection vs. surgical resection + WBRT

- Surgical resection \pm WBRT or local RT vs. stereotactic radiosurgery (SRS) \pm WBRT or local RT

- Surgical resection vs. surgical resection + SRS

- Surgical resection + WBRT vs. surgical resection + SRS

Should patients with newly diagnosed metastatic brain tumors undergo SRS compared with other treatment modalities?

Comparisons include:

- WBRT vs. WBRT + SRS

- SRS vs. WBRT + SRS

- SRS vs. WBRT

- SRS \pm WBRT or local RT vs. surgical resection \pm WBRT or local RT

$-\mathrm{SRS} \pm$ surgical resection vs. WBRT \pm surgical resection

- Single session SRS \pm WBRT vs. fractionated SRS \pm WBRT

Should patients with newly diagnosed metastatic brain tumors be given chemotherapy for the treatment of brain metastases compared with no chemotherapy?

Comparisons include:

Any comparative studies evaluating chemotherapy alone or in combination with other treatment modalities for the treatment of newly diagnosed brain metastases

upon and these correspond to the eight practice guideline papers in this series. Four of the questions specifically focus on the treatment of patients with newly diagnosed brain metastases (Table 1), one question addresses the treatment of recurrent/progressive metastatic brain disease (Table 2) and the remaining three questions are relevant to all patients with brain metastases (Table 3). Some of the questions had several parts.
Table 2 Guideline questions specifically addressing treatment of patients with recurrent and/or progressive brain metastases

Patients with recurrent or progressive brain metastases

What evidence is available regarding the use of WBRT, SRS, surgical resection or chemotherapy for the treatment of recurrent and/or progressive brain metastases?

- Any study evaluating the use of WBRT, SRS, surgical excision or chemotherapy alone or in combination in patients with recurrent and/or progressive brain metastases

If WBRT is used, what impact does tumor histopathology have on treatment outcomes?

- Any study evaluating the outcome(s) of WBRT by tumor histopathology (e.g., radiation-sensitive tumors vs. radiationresistant tumors) in patients with recurrent and/or progressive brain metastases

Table 3 Guideline questions addressing all patients with brain metastases

For all patients with brain metastases

Do prophylactic anticonvulsants decrease the risk of seizure in patients with metastatic brain tumors compared with no treatment?

Comparison: anticonvulsant prophylaxis vs. none

Do steroids improve neurologic symptoms in patients with metastatic brain tumors compared to no treatment? If steroids are given, what dose should be used?

Comparisons include:

- Steroid therapy vs. none

- Comparison of different doses of steroid therapy

What evidence is available regarding the following emerging/ investigational therapies for the treatment of metastatic brain tumors?

New radiation sensitizers:

Motexafin-gadolinium

RSR 13

Local modalities placed at the time of surgical excision:

Local irradiation: gliasite radiation therapy system/balloon placement

Local chemotherapy: BCNU-impregnated polymers

Brachytherapy

New chemotherapeutic agents:

Temozolomide

Fotemustine

Molecular targeted agents:

Gefitinib (ZD1839)

Anti-angiogenesis agents:

Bevacizumab (Avastin)

For the purposes of the systematic review and the clinical practice guidelines, brain metastases have been defined as solid metastases to the brain from systemic cancer. The definition excludes leptomeningeal metastatic disease. 


\section{Literature search strategy}

A broad literature search strategy was undertaken to identify all citations relevant to the management of brain metastases. The following electronic databases were searched from 1990 to September 2008: MEDLINE $^{\circledR}$, Embase $^{\circledR}$, Cochrane Database of Systematic Reviews, Cochrane Controlled Trials Registry, and Cochrane Database of Abstracts of Reviews of Effects. The search strategy used a combination of subheadings and text words. The specific search terms used for MEDLINE $^{\circledR}$ are provided in Appendix A; this search strategy was modified accordingly for appropriate terms for each database searched. Reference lists of included studies were also screened for potentially relevant studies.

An additional electronic database search was conducted to identify randomized trials published from 1970 forward that evaluated different dose/fractionation schedules of whole-brain radiation for the treatment of brain metastases. This was done to capture seminal studies in this area known to have been undertaken in this earlier time frame. The initial electronic search commenced at 1990 given the lack of any known comparative data for radiation, surgical resection and radiosurgery prior to this date, although additional searches were conducted as far back as 1970 as outlined above.

Conference proceedings from the 2006-2008 meetings of the AANS, CNS, Society for NeuroOncology (SNO), American Society for Therapeutic Radiation and Oncology (ASTRO), American Society for Clinical Oncology (ASCO) and the AANS/CNS joint section on tumors satellite symposiums were searched for abstracts eligible for inclusion in the emerging and investigational therapies guideline question.

\section{Study selection, quality assessment and statistical methods}

The search of the bibliographic databases identified 16,966 possibly relevant citations. These citations were screened in duplicate using an online systematic review management system designed by TrialStat! (http://www.ESRNEXUS. com). Two independent reviewers evaluated titles and abstracts using a priori eligibility criteria in standardized forms. Cases of disagreement were resolved by a third reviewer. The same process was applied to full text screening of potentially relevant studies. Articles that met the eligibility criteria for one or more of the questions were grouped according to the questions they addressed. Reasons for exclusion were documented. The eligibility criteria for each of the eight questions are documented in the individual clinical practice guideline papers in this series.
Table 4 Management of brain metastases guideline panel

\begin{tabular}{ll}
\hline Guideline panel members & Affiliations \\
\hline Steven N. Kalkanis, MD, & $\begin{array}{l}\text { Dept. of Neurosurgery, } \\
\text { Chair }\end{array}$ \\
Henry Ford Health System, Detroit, MI \\
Mario Ammirati, MD, & Dept. of Neurosurgery, \\
MBA & Ohio State University Medical Center, \\
& Columbus, OH \\
David W. Andrews, MD & Dept. of Neurosurgery, \\
& Thomas Jefferson University, \\
& Philadelphia, PA \\
Anthony L. Asher, MD, & Dept. of Neurosurgery, \\
FACS & Carolina Neurosurgery and Spine \\
& Associates, Charlotte, NC \\
Stuart H. Burri, MD & Dept. of Radiation Oncology, \\
& Carolinas Medical Center, Charlotte, \\
\end{tabular}

Charles S. Cobbs, MD

Dept. of Neurosciences,

California Pacific Medical Center, San Francisco, CA

Laurie E. Gaspar, MD

Dept. of Radiation Oncology,

University of Colorado-Denver, Denver, CO

Douglas Kondziolka, MD, $\mathrm{MSc}$

Dept. of Neurological Surgery,

University of Pittsburgh Medical Center, Pittsburgh, PA

Mark E. Linskey, MD

Dept. of Neurosurgery,

University of California-Irvine Medical Center, Orange, CA

Jay S. Loeffler, MD, FACR Dept. of Radiation Oncology,

Massachusetts General Hospital, Boston, MA

Michael McDermott, MD

Dept. of Neurosurgery,

University of California San Francisco, San Francisco, CA

Minesh P. Mehta, MD

Dept. of Human Oncology,

University of Wisconsin School of Medicine, Madison, WI

Tom Mikkelsen, MD Dept. of Neurology,

Henry Ford Health System, Detroit, MI

Jeffrey J. Olson, MD

Dept. of Neurosurgery,

Emory University School of Medicine, Atlanta, GA

Nina A. Paleologos, MD Dept. of Neurology,

Northshore University Health System, Evanston, IL

Roy A. Patchell, MD

Dept. of Neurology,

Barrow Neurological Institute, Phoenix, AZ

Timothy Charles Ryken, MD, MS, FACS

Dept. of Neurosurgery,

Iowa Spine and Brain Institute, Iowa City, IA 
Studies which met the eligibility criteria were data extracted by one reviewer and the extracted information was checked by a second reviewer. The PEDro scale $[2,3]$ was used to evaluate the methodological quality (internal validity) of randomized trials. The quality of comparative studies using non-randomized designs was evaluated using eight items selected and modified from existing scales (Appendix B).

Evidence and summary tables, reporting the extracted study information and quality assessment, were generated for all of the included studies for each of the eight questions. Meta-analyses were undertaken when sufficient data for pooling was available for the outcomes of interest. In only one of the eight guideline papers in this series was the criteria for pooling met. The specific details of the metaanalyses undertaken for this question are provided in the whole-brain radiation therapy (WBRT) guideline paper by Gaspar et al. [4].

\section{Evidence classes and levels of recommendations endorsed by the AANS/CNS}

Both the evidence classification and the strength of the recommendations were graded according to the criteria endorsed by the AANS/CNS (Table 5). The class of evidence assigned to each study was based on study design alone (i.e., class I, II, or III). The strength of the

Table 5 AANS/CNS evidence classes and levels of recommendation

\section{Evidence classification}

Class I Evidence provided by one or more well-designed randomized controlled clinical trials, including overview (meta-analyses) of such trials

Class II Evidence provided by well-designed observational studies with concurrent controls (e.g. case control and cohort studies)

Class III Evidence provided by expert opinion, case series, case reports and studies with historical controls

\section{Levels of recommendation}

Level 1 Generally accepted principles for patient management, which reflect a high degree of clinical certainty (usually this requires Class I evidence which directly addresses the clinical questions or overwhelming Class II evidence when circumstances preclude randomized clinical trials)

Level 2 Recommendations for patient management which reflect clinical certainty (usually this requires Class II evidence or a strong consensus of class III evidence)

Level 3 Other strategies for patient management for which the clinical utility is uncertain (inconclusive or conflicting evidence or opinion) recommendations made (i.e., level 1,2 , or 3 ) took into account aspects of study quality, not just study design. Specifically, the level of a recommendation made could be decreased, based on consensus input, if there were methodological concerns regarding the studies that provided evidence for that particular recommendation.

\section{Guideline panel consensus and practice guideline approval process}

Small writing groups composed of four to eight members of the clinical guideline panel were assigned to each of the eight questions, and each question had at least one member from each subspecialty as part of the initial writing team. Each group was provided with the included studies and the evidence/summary tables for their specific question, as well

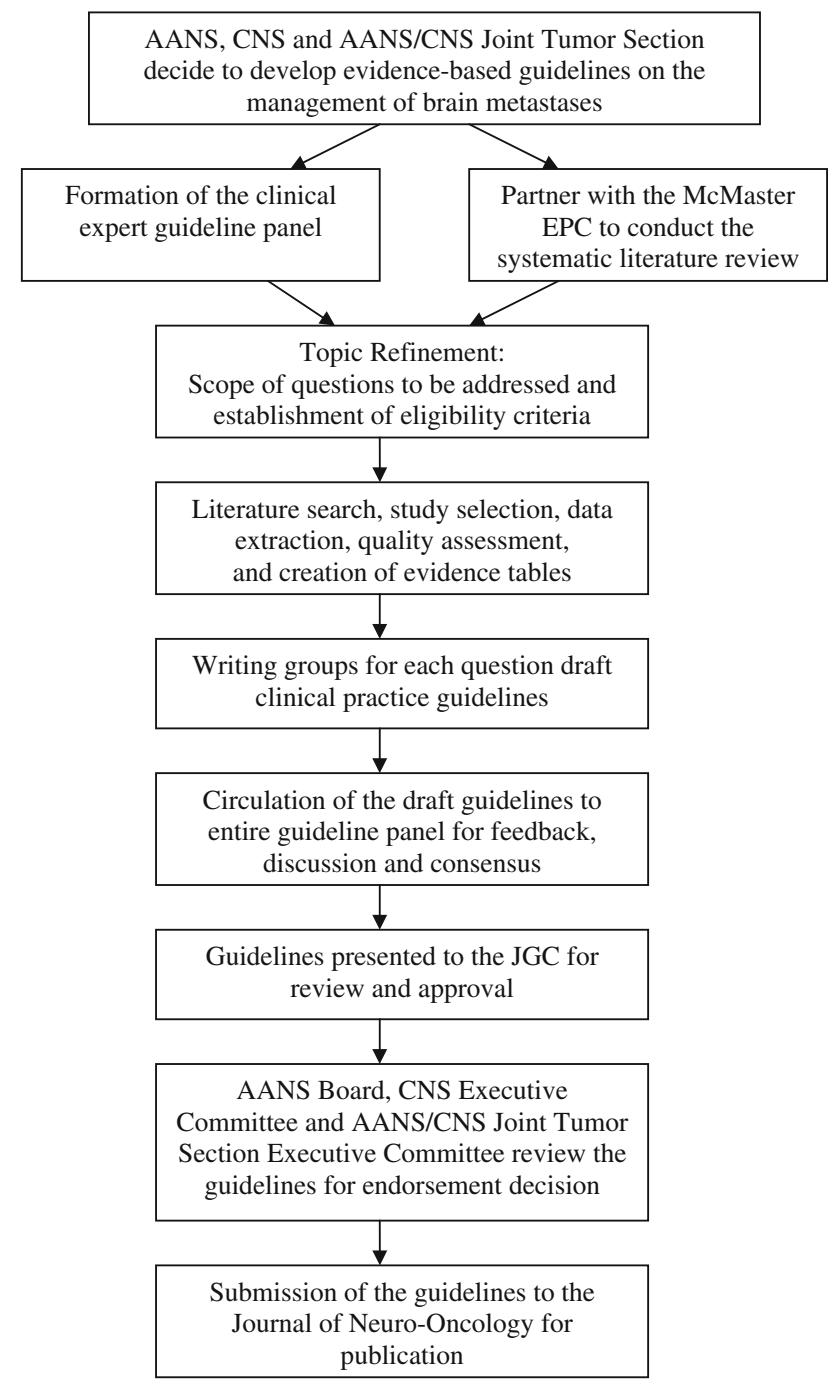

Fig. 1 Overview of the guideline development process 
as an exhaustive statistical analysis, from the McMaster EPC team. Using this information, the small writing groups were responsible for drafting the clinical practice guideline for their respective questions. The draft guidelines were then circulated to the entire clinical guideline panel for feedback, discussion, and ultimately approval.

In accordance with the initial goal of a 1-year time horizon, the completed evidence-based clinical practice guidelines on the management of brain metastases were presented to the Joint Guidelines Committee (JGC) of the AANS/CNS for approval approximately 12 months after starting the evidence review process. As part of their approval process, the JGC could provide input on the content of the clinical practice guidelines. Once approved by the American Association of Neurological Surgeons and the Congress of Neurological Surgeons, the guidelines were also presented to the executive leadership of the Society for Neuro-oncology (SNO), the American Society for Radiation Oncology (ASTRO), in addition to the AANS/CNS Joint Tumor Section, to offer multidisciplinary review and endorsement with plans for online publication and dissemination in all of the various organized societies concerned with the treatment of brain metastases.

Development of this set of evidence-based clinical practice guidelines was editorially independent from the funding agencies. The funding agencies (AANS Board, CNS Executive Committee, and AANS/CNS Joint Tumor Section Executive Committee) review of these guideline papers, following JGC approval but prior to submission for publication, was limited to whether or not to endorse or reject this body of work. Figure 1 provides an outline of the key steps in the process of developing these clinical practice guidelines.

\section{Plans for updating the guidelines}

Searches of a clinical trial registry (clinicaltrials.gov) identified several on-going randomized trials that are evaluating treatment comparisons directly addressed by questions in this guideline series. The outcome of these trials will be monitored and updates to the relevant guidelines will be undertaken as new evidence develops and as clinical need indicates.

Acknowledgments We would like to acknowledge the contributions of the McMaster Evidence-based Practice Center (EPC), Dr. Parminder Raina (Director). Dr. Lina Santaguida (Co-Associate Director, Senior Scientist) led the EPC staff. We would like to acknowledge the following EPC staff, Maureen Rice, Librarian, Rachel Morris, Research Assistant, and Mary Gauld, Senior Research Manager. The EPC staff were responsible for managing the systematic review process, searching for and retrieving, reviewing, data abstraction of all articles, preparation of the tables and the formatting and editing of the final manuscripts.

Disclaimer of liability The information in these guidelines reflects the current state of knowledge at the time of completion. The presentations are designed to provide an accurate review of the subject matter covered. These guidelines are disseminated with the understanding that the recommendations by the authors and consultants who have collaborated in their development are not meant to replace the individualized care and treatment advice from a patient's physician(s). If medical advice or assistance is required, the services of a competent physician should be sought. The proposals contained in these guidelines may not be suitable for use in all circumstances. The choice to implement any particular recommendation contained in these guidelines must be made by a managing physician in light of the situation in each particular patient and on the basis of existing resources.

Disclosures All panel members provided full disclosure of conflicts of interest, if any, prior to establishing the recommendations contained within these guidelines.

Open Access This article is distributed under the terms of the Creative Commons Attribution Noncommercial License which permits any noncommercial use, distribution, and reproduction in any medium, provided the original author(s) and source are credited.

\section{Appendix A: Medline search strategy}

See Table 6.

Table 6

Search strategy used for the Medline database

1. exp Brain Neoplasms/

2. Central Nervous System Neoplasms/

3. or/1-2

4. (metasta $\$$ or secondary).tw.

5. exp Neoplasm Metastasis/

6. or/4-5

7. 3 and 6

8. exp Brain Neoplasms/sc [Secondary]

9. ((metasta $\$$ or secondary) adj3 (brain or cereb\$ or intercranial)).tw.

10. or/8-9

11. 7 or 10

12. animals/not (animals/and humans/)

13. 11 not 12

14. limit 13 to $\mathrm{yr}=$ "1990-2008"

15. limit 14 to english language

\section{Appendix B: Quality assessment criteria}

See Table 7. 


\section{Table 7}

Quality assessment of randomized studies using the PEDro

criteria [2, 3]

1. Eligibility criteria specified?

2. Random allocation?

3. Allocation concealed?

4. Groups similar at baseline on most important prognostic indicators?

5. Subjects blinded to treatment?

6. Blinding of clinicians who administered treatment?

7. Blinding of assessors who measured at least 1 key outcome?

8. Measures of at least 1 key outcome from more than $85 \%$ subjects initially allocated to groups?

9. All subjects for whom outcome measures were available received the treatment or control condition as allocated or data was analyzed by "intention to treat"?

10. Results of between-group statistical comparisons are reported for at least one key outcome?

11. Study provides both point measures and measures of variability for at least 1 key outcome?

Quality assessment of non-randomized studies

1. Are the respective study arms comparable on the basis of the design or analysis?

2. Is the selection of the study arms appropriate?

3. Were the eligibility criteria the same for all of the study arms?

4. Does the study report whether or not participants received additional interventions that may influence the results (e.g. additional treatment for recurrent/progressive brain metastases)?

5. Was there adequate adjustment for confounding in the analyses from which the main findings were drawn?

6. Are the interventions of interest clearly described?

7. Were the study subjects in different intervention groups recruited over the same period of time?

8 . Is the follow-up of the groups adequate?

\section{References}

1. Gavrilovic IT, Posner JB (2005) Brain metastases: epidemiology and pathophysiology. J Neurooncol 75(1):5-14

2. Centre for Evidence-Based Physiotherapy (2009) Physiotherapy evidence database (PEDro). http://www.pedro.org.au/. Accessed Jan 2009

3. Maher CG, Sherrington C, Herbert RD, Moseley AM, Elkins M (2003) Reliability of the PEDro scale for rating quality of randomized controlled trials. Phys Ther 83(8):713-721

4. Gaspar LE, Mehta MP, Patchell RA, Burri SH, Robinson PD, Morris RE et al (2009) The role of whole brain radiation therapy in the management of newly diagnosed brain metastases: a systematic review and evidence-based clinical practice guideline. J Neurooncol. doi:10.1007/s11060-009-0060-9 